\title{
Haploidentical transplantation in pediatric non-malignant diseases: A retrospective analysis on behalf of the Spanish Group for Hematopoietic Transplantation (GETH)
}

juan torres canizales ${ }^{1}$, Cristina Ferreras ${ }^{2}$, Antonia Pascual ${ }^{3}$, Laura Alonso ${ }^{4}$, Alexandra Regueiro $^{5}$, Mercedes Plaza ${ }^{6}$, JOSE MARIA PEREZ-HURTADO ${ }^{7}$, Ana Benito ${ }^{8}$, Jose Couselo $^{9}$, José Luis Fuster Soler ${ }^{10}$, Miguel Blanquer Blanquer ${ }^{10}$, Mariana Diaz-Almirón ${ }^{11}$, David Bueno ${ }^{11}$, Yasmina Mozo ${ }^{11}$, Alicia Gómez ${ }^{12}$, José Luis Vicario ${ }^{13}$, Antonio Balas ${ }^{14}$, Luisa Sisinni ${ }^{11}$, Cristina Díaz de Heredia ${ }^{4}$, and Antonio Pérez-Martínez ${ }^{15}$

${ }^{1}$ hospital la paz

${ }^{2}$ La Paz University Hospital Biomedical Research Foundation

${ }^{3}$ Hospital Regional Universitario Carlos Haya

${ }^{4}$ Hospital Universitari Vall d'Hebron

${ }^{5}$ Hospital Clinico Universitario de Santiago de Compostela

${ }^{6}$ Virgen de la Arrixaca University Hospital

${ }^{7}$ HOSPITAL UNIVERSITARIO VIRGEN DEL ROCIO

${ }^{8}$ Hospital Universitario de Salamanca

${ }^{9}$ University of Santiago Clinical Hospital

${ }^{10}$ Hospital Clinico Universitario Virgen de la Arrixaca

${ }^{11}$ Hospital Universitario La Paz

${ }^{12}$ Universidad Autonoma de Madrid

${ }^{13}$ Centro de Transfusión de Madrid

${ }^{14}$ Centro de Transfusión de la Comunidad de Madrid

${ }^{15}$ HOSPITAL UNIVERSITARIO LA PAZ

June 22, 2020

\begin{abstract}
Background: We retrospectively analyzed the data of children with non-malignant diseases who have received a haploidentical hematopoietic stem cell transplant (haplo-HSCT). A total of 31 haplo-HSCT were performed in 26 pediatric patients using ex vivo T cell-depleted (TCD) graft platforms or post-transplantation cyclophosphamide (PT-Cy) from January 2001 to December 2016 in 7 Spanish centres. Procedure: A total of five cases were unmanipulated PT-Cy haplo-HSCT, sixteen received highly purified CD34+ cells, ten were ex vivo TCD graft manipulated either with CD3+CD19+ depletion $(\mathrm{n}=1), \mathrm{TCP} \alpha \beta+\mathrm{CD} 19+$ selection $(n=7)$ or naive CD45RA $+T$ cells depletion $(n=2)$. Peripheral blood stem cells were the only source in patients following TCD haplo-HSCT, and bone marrow was the source for one PT-Cy haplo-HSCT. The most common indications for transplant were primary immune deficiency disorders (PIDs) 18, severe aplastic anemia (SAA) 4, osteopetrosis 2 and thalassemia 2. Results: The 1-year cumulative incidence of graft failure was $27.4 \%$. The 1-year III-IV acute graft versus host disease (aGvHD) and 1-year chronic graft versus host disease (cGvHD) were $34.6 \%$ and $16.7 \%$ respectively. Besides, the 2-year overall survival (OS) and the 2-year GvHD-free and relapse-free survival (GRFS) were $44.9 \%$ for PIDS and $37.6 \%$ for the other NMDs. The TRM at day 100 was $30.8 \%$. Conclusions : These results are discouraged and need to be improved to offer a guaranteed
\end{abstract}


treatment for these patients. Improvements will come if procedures are centralized in centres of expertise. The decision between T-cell depletion platforms will depend on the patients' underlying diseases, comorbidities, and conditioning regimens.

\section{INTRODUCTION}

Haploidentical stem cell transplantation (haplo-HSCT) is a feasible option for a cure in children with both malignant and non-malignant diseases (NMDs) such as bone marrow failure syndromes, inborn errors of metabolism (IEM) and hemoglobinopathies ${ }^{1-4}$ where no matched related or unrelated donor is available. This procedure has achieved engraftment rates of $70 \%$, durable graft of $67 \%$ and overall survival (OS) of $60 \%{ }^{4}$.

The advantages of haploidentical donors include immediate and almost universal availability, avoidance of unrelated donor search costs, and the availability for potential post-transplantation cellular therapy ${ }^{5,6}$. Recent data from the European Society of Blood and Marrow Transplantation (EBMT), reported an increased by $22 \%$ and $24.5 \%$ in allogeneic HSCT for PIDs and IEM and stabilization for thalassemia ${ }^{6-8}$.

Despite the tremendous advances toward the clinical feasibility of haplo-HCT there are still unsolved obstacles to the success of the procedure, such as graft rejection, delayed recovery of adaptive immunity, and increased risk of severe infections and transplantation-related mortality (TRM) compared with human leukocyte antigen (HLA)-matched unmanipulated allografts ${ }^{9,10}$. This challenge is reflective of the need for extensive T-cell depletion and immunosuppression to control graft versus host disease (GvHD) on one hand, and facilitation of engraftment, immune reconstitution, protection from infections, and prevention of relapse on the other hand ${ }^{11}$.

Several strategies have been developed to overcome the high rates of GvHD and graft failure obtained from early attempts at using $\mathrm{T}$ cell replete grafts from haploidentical donors using conventional preparative regimens ${ }^{5}$. Recent technological advances of $\mathrm{T}$ cell depletion have made this form of HSCT accessible and feasible $^{12,13}$. Two main platforms of haplo-HSCT have been developed in the clinical setting aimed at reducing the morbidity and mortality associated with GvHD. First, ex vivo total or partial T cell-depleted (TCD) grafts, and in recent years, T cell-replete grafts followed by post-transplantation cyclophosphamide $(\mathrm{PT}-\mathrm{Cy})^{14}$.

Data from different groups using partial TCD grafts with $\mathrm{TCR} \alpha \beta^{+} \mathrm{CD} 19^{+}$depletion and replete graft followed PT-Cy reported excellent survival in PID and thalassemia ${ }^{15}$. Another way to purge total $\mathrm{T}$ cells is depletion of $\mathrm{CD}^{+}$and $\mathrm{CD} 19^{+}$lymphocytes, which allows other cell types to be retained in the donor graft, such as natural killer (NK) cells, dendritic cells, and monocytes. Despite optimal engraftment, alloreactive lymphocytes retained in the graft increased GvHD even with GvHD pharmacological prophylaxis ${ }^{16-18}$. The current platforms of partial $\mathrm{T}$ cell depletion, such as $\alpha \beta^{+} \mathrm{T}$ cells and naïve $45 \mathrm{RA}^{+} \mathrm{T}$ cells, have optimized the initial CD34 ${ }^{+}$selection procedure limitations, keeping some T cell subsets such as $\gamma \delta^{+} \mathrm{T}$ cells, NK cells and memory $\mathrm{T}$ cells without increasing $\mathrm{GvHD}^{19,20}$.

Compared to match sibling donors (MSD), haplo-HSCT donors have a trend to reduce OS $^{21,22}$, but still, data on NMD are scarce. Different groups have reported a 1 or 2 year OS rate of $60-80 \%$ in a group of NMD using TCD or PT-Cy with low rates of GvHD and improved immune reconstitution using an ex vivo $\alpha \beta^{+}$TCD platform and B cell depletion ${ }^{23-25}$, and haplo-HSCT PT-Cy ${ }^{26,27}$. Consistent results have been reported from our group, the Spanish Working Group for Hematopoietic Transplantation (GETH), in the largest analysis of clinical outcomes in children comparing both platforms in pediatric hematological malignancies. The study showed a 2-year relapse-free and graft versus host disease free of $49.2 \%$ and $40.4 \%$ respectively, being relapse the main cause of haplo-HSCT failure ${ }^{28}$.

This retrospective and multicenter study aimed to analyze the feasibility and outcome of a Spanish cohort of haplo-HSCT in children with NMD and propose a common future strategy.

\section{METHODS}




\section{Patients}

We report a retrospective analysis of 31 haplo-HSCT performed in 26 children with NMD in 7 hospital institutions from Spain from January 2001 to December 2016 (Table S1). The Spanish Working Group for Bone Marrow Transplantation in Children (GETMON) requested that all centers obtained a completed data from all pediatric patients undergoing a haplo-HSCT treatment for NMD. Written informed consent was obtained from the patients' parents following the Declaration of Helsinki. Transplant indication was performed according to International Guidelines for NMDs and performed according to each approved institutional protocol. As a rule, the exclusion criterion was poor performance status (Lansky score $<60 \%$ ).

\section{Stem cell source, donor selection and graft manipulation}

The haploidentical donor was selected based on availability and their preference among the first-degree relatives. Stem cells were obtained from granulocyte colony-stimulating factor-mobilized peripheral blood $(\mathrm{n}=30)$ and bone marrow $(\mathrm{BM})(\mathrm{n}=1)$. Transplantation was avoided if anti-HLA antibodies were present in significant titers (IgG >10.000 MFI; C3d >1000 MFI). In 5 of the 31 cases who received haplo-HSCT the graft was unmanipulated and PT-Cy was used as GvHD prophylaxis. In the remaining 26 cases, the ex vivoTCD graft was performed as follows: in 16 cases, peripheral blood stem cells (PBSCs) were highly purified for CD34 ${ }^{+}$cells; in 1 case, patients received a combined depletion of donor $\mathrm{CD}^{+}{ }^{+} \mathrm{T}$ cells and CD19 ${ }^{+}$ $\mathrm{B}$ cells; in 7 cases, patients received a combined depletion of $\alpha \beta^{+} \mathrm{T}$ cells and CD19+ $\mathrm{B}$ cells; and in 2 cases, patients received a highly purified $\mathrm{CD} 34^{+}$graft, adding to the graft a product depleted in naïve CD45RA ${ }^{+}$ T cells, using the CliniMACS System as previously described ${ }^{29}$.

\section{Conditioning regimen and graft-versus-host disease prophylaxis}

Conditioning regimens used were individualized and modified as per the underlying disorder and type of $\mathrm{T}$ cell depletion used as illustrated in Table 2. The conditioning regimens were performed according to international guidelines for NMDs that differed according to the original disorder and based on the combination of fludarabine (90-150 mg/m2), melphalan (100-140 mg/m2), thiotepa (10 mg/kg) and serotherapy or TBI (2-4 Gy). Reduced-intensity conditioning (RIC) was used in 18 patients, by the combination of fludarabine $(150-160 \mathrm{mg} / \mathrm{m} 2)$ plus either busulfan $(6.4 \mathrm{mg} / \mathrm{kg})$, melphalan, thiotepa or treosulfan $(42 \mathrm{~g} / \mathrm{m} 2)$. Seventeen patients received serotherapy, fifteen with anti-thymocyte globulin (ATG) and two received alemtuzumab. ATG $(15 \mathrm{mg} / \mathrm{kg}$ total $)$ was given over days -5 to -3 , and alemtuzumab $(1 \mathrm{mg} / \mathrm{kg}$ total $)$ was given over days -8 to -4 . GvHD prophylaxis strategies are described in Table 2. Cyclosporine (CsA), mycophenolate mofetil (MMF), methotrexate, tacrolimus or sirolimus were used at least 6 months after HSCT, respectively, unless required longer for GvHD. For the prevention of cyclophosphamide-related hemorrhagic cystitis, intravenous mesna was administered at days +3 and +4 (total daily dose of $40 \mathrm{mg} / \mathrm{kg}$ ). The aGvHD was clinically graded according to modified Glucksberg criteria ${ }^{30}$. The cGvHD was diagnosed based on at least 1 diagnostic clinical sign of cGvHD according to National Institutes of Health criteria ${ }^{31}$ or 1 distinctive manifestation confirmed by biopsy.

\section{Engraftment and chimerism}

Primary graft failure was defined as the absence of donor-derived myeloid cells at day +30 or reconstitution with autologous cells, and secondary graft failure as a sustained drop in the absolute neutrophil count (ANC) to $0.5 \times 10^{9} / \mathrm{L}$ for [?] 5 days. Chimerism analysis was performed based on short tandem repeat microsatellite regions (sensitivity 1\% to 3\%). For each donor-recipient pair, DNA was isolated using the QIAamp Blood Kit (Qiagen, Chatsworth, CA, USA), and informative alleles were identified for a panel of 11 short tandem repeats, using the ABI Prism 3130x1 System (Applied Biosystems, Foster City, CA, USA) and GeneScan 4,0 Software (Applied Biosystems). The percentage of donor alleles was calculated at least monthly for the first three months and quarterly thereafter until the year, continuing if mixed chimerism was present.

\section{Infections and supportive care}

Viral surveillance was performed weekly for cytomegalovirus (CMV), EBV, and adenovirus as guidelines recommended. Given that this was a retrospective multicenter study with a large cohort, we only collected 
infectious complications considered clinically relevant by the local HSCT team. All patients received antimicrobial prophylaxis for bacteria, fungal, Pneumocystis jiroveci, and herpes infection. The patients received supportive care treatment according to the standard-of-care and institutional practices.

\section{Immune reconstitution}

When it was available the phenotyping of immune cells $\mathrm{T}$ lymphocytes $\left(\mathrm{CD} 3^{+}\right)$, $\mathrm{T}$ cell subsets $\left(\mathrm{CD} 4^{+}\right.$ and $\left.\mathrm{CD} 8^{+}\right)$, NK $\left(\mathrm{CD} 3^{-} \mathrm{CD} 56^{+}\right)$cells and $\mathrm{B}$ lymphocytes $\left(\mathrm{CD} 19^{+}\right)$was performed after density-gradient centrifugation of fresh peripheral blood (Ficoll-Paque, GE Healthcare, South Burlington, VT, USA). A multiparametric flow cytometry analysis was performed in each center at 30,60, 90, 180 and 360 days after transplantation.

\section{Statistical analyses}

Quantitative variables were expressed as median and either range or interquartile range (IQR) and qualitative variables as frequency and percentage. The cumulative incidence of, graft failure, aGvHD and cGvHD were estimated, considering deaths without any of these events as competing risk as described by Gray previously ${ }^{33}$. The major study endpoints were overall survival (OS), and TRM at day +100 . The TRM was defined as death after haplo-HSCT without disease progression. Patients were censored at the time of death or last follow-up. The OS was defined as the time from transplantation to death from any cause. Moreover, we considered the variable GvHD-free, relapse-free survival (GRFS), in which events include grades III-IV aGvHD, severe cGvHD, or death post haplo-HSCT associated with progression of the underlying disease ${ }^{32}$. The OS and GRFS rates were calculated using the Kaplan-Meier product-limit method including standard error (SE) and log-rank test for the univariate analysis. All statistical tests were 2 -sided, and $\mathrm{P}$-value $<0.05$ was considered statistically significant. The statistical analyses were performed with SPSS software (version 17.0; SPSS Inc., Chicago, IL, USA).

\section{RESULTS}

Patient characteristics, source, graft and engraftment

Table 1 shows the main characteristics of the patients undergoing haplo-HSCT. Three patients received haplo-HSCT as a rescue procedure from a previous allogeneic HSCT. The overall median age at transplant was 2.11 years old, IQR 9.14, $(\mathrm{PID}=1.77$, IQR 6.87 vs other $\mathrm{NMD}=5.22$, IQR 8.56, $\mathrm{p}=\mathrm{ns})$. The median donor age was 41 years old (IQR 14). PBSCs were exclusively used for ex vivo TCD haplo-HSCT and 1 of 5 PT-Cy administrations used BM as a stem cell source. The median number of CD34 ${ }^{+}$cells infused was $10 \times 10^{6} / \mathrm{kg}$ (IQR 9.71). A total of 6 patients (5 PID and 1 SAA) developed graft failure to the first haplo-HSCT transplant procedure, and 5 of them were re-transplanted (Table S2). The 1-year cumulative incidence of graft failure was $27.4 \%(\mathrm{SE}=0.126)$ (Table 1 and Figure 1). We did not find any factors associated with graft failure in the univariate analyses.

\section{Acute and chronic graft-versus-host disease and major infection complications and transplant- related mortality}

Table 2 shows in detail the data on GvHD and HSCT outcomes. The 1-year cumulate incidence rate of aGvHD grades I-II was 19.9\% ( $\mathrm{SE}=0.119), 34.6 \%(\mathrm{SE}=0.147)$ for grade III-IV aGvHD and $16.7 \%(\mathrm{SE}=0.108)$ for cGvHD (Table 1). After transplant, infections were detected in $23(74 \%)$ of patients, mainly caused by bacterial in $38 \%(\mathrm{n}=10)$, viral in $42 \%(\mathrm{n}=11)$ and fungal infections in $14 \%(\mathrm{n}=4)$ of cases. Table 2 shows the causes of death in all series. The TRM at day 100 was $30.8 \%(\mathrm{SE}=0.091)$ (Table 1).

Overall survival, disease-free survival, cumulative relapse incidence, and graft versus host disease-free/relapse-free survival

The follow-up time was 828 days varying from 212 to 5079 days. The 2-year OS was $44.9 \%$ ( $\mathrm{SE}=0.100),(50 \%$ in other NMDs vs $43 \%$ in PIDs, $\mathrm{P}=$ n.s.) (Table 1). The overall 2-year GRFS after transplant was $37.6 \%$ $(\mathrm{SE}=0.097),(37.0 \%$ in PIDs vs $37.5 \%$ in other NMDs, $\mathrm{P}=$ n.s.). There were no significant differences in the OS and GRFS in patients who received the first haplo-HSCT versus patients with a previous transplant 
(Figure 1). We did not find any factors associated with OS or GRFS in the univariate analysis. Chimerism data were available in 22 of 26 patients (Table 2). Data showed complete donor chimerism at day +30 in 19 of patients. One PID patient had mixed chimerism beyond 2 years after haplo-HSCT (autologous polymorphonuclear neutrophils below $5 \%$ and $\mathrm{CD}^{+} 40 \%$ ) but without clinical significance.

\section{Immune reconstitution}

We were only able to collect data from 8 surviving patients. First, we observed an increase of NK cells at day +30 to 74 cells/ $\mu$ IQR 21 , rising at day +90 to 224 cells/ $\mu$ l, IQR 235 and remaining stable until day +360 to 187 cells/ $\mu \mathrm{l}$, IQR 176 . The CD8 ${ }^{+} \mathrm{T}$ cell subset increased from 120 cells/ $\mu \mathrm{l}$, IQR 32 at day +60 , continuing to 358 cells/ $\mu$ l, IQR 260 at day +90 , and 795 cells/ $\mu$ l, IQR 255 at day +180 , and 709 cells $/ \mu$, IQR 1506 at day +360 . In addition, the gradual increase in $\mathrm{CD} 4^{+} \mathrm{T}$ cell subset was observed from day +90 to 120 cells/ $\mu$ l, IQR 197, followed on days +180 to 207 cells/ $\mu$ l, IQR 483 and at day +360 to 562 cells/ $\mu$, IQR 278. Finally, the increase of B-lymphocytes CD19+ was detected from day +180 to 168 cells/ $\mu$ l, IQR 249 and at day +360 to 198 cells/ $\mu$ l, IQR 475 , but remained below to the normal range for that age. The median lymphocytes per diagnosis group are represented in Figure S1.

\section{DISCUSSION}

This study reports the accumulated experience of all Spanish centers performing haplo-HSCT in the pediatric population with NMDs, which corresponds to $13.9 \%$ of the cumulative activity of haplo-HSCT ${ }^{28}$. In our study, the 2-year OS of $44.9 \%$ was lower compared to our reported series on malignant disorders (2-year $\mathrm{OS}=55.1 \%{ }^{28}$, and recently reported from different groups in NMDs, which also differs according to the diagnosis $2,12,34-39$. These differences can be explained because the use of haplo-HSCT in NMDs was not a routine practice in the first decade of this study (2001-2010), being MSD the first alternative in both PIDs and other NMDs. Additionally, the following ex vivo TCD graft alternative and its availability are only possible in centers where it is a routine procedure. One reason for the low OS could be that about $50 \%$ of the grafts were CD $34^{+}$ex vivo TCD. It is well known that CD34 ${ }^{+}$selection in the haploidentical setting is a risk for graft failure and delayed immune reconstitution ${ }^{6}$. Until 2012 the CD34 ${ }^{+}$selection was the only haplo-HSCT in our centers. Graft failure in our cohort was an important undesirable event and re-transplant only was successful in one patient. aGvHD grade III-IV was also higher as expected. Therefore both, graft failure and GvHD impacted in the short and long term of HSCT, increasing TRM, and they should be taken into account in the alternative transplant procedure selection process for each particular case ${ }^{40}$.

Also, as haplo-HSCT is not the first treatment option, it should be noted that the patients in our series had a major risk factor for a poor post-HSCT outcome, in most cases may take time between diagnosis and transplantation, leading to increase cumulative morbidity, mostly in the case of PID patients due to infections, which contribute to high post-HSCT mortality ${ }^{21,41,42}$. In our cohort, most of SCID patients received RIC without receiving GvHD prophylaxis, and three SCID patients did not receive conditioning. Despite this strategy, up to 4 of 11 SCID patients developed GvHD. The timing of the transplant is especially important in SCID patients, and the best outcome is achieved when the patients are transplanted before they developed infectious complications and end-organ damage ${ }^{2}$. In non-SCID diseases, conditioning is always required for all donor sources. HLA-disparity necessitates deep T-cell depletion to avoid GvHD ${ }^{1}$. Although our non-SCID patient group is heterogeneous, the use of TCD platforms did not improve the survival outcomes; therefore, either the combination of ATG, the conditioning or GvHD prophylaxis in these patients may lead to a delay in immune reconstitution that increases the risk of mortality.

Although PT-Cy data are scarce ${ }^{39,43,46}$, published data suggest that this treatment option could be an alternative to improve GvHD rates and increase survival in NMD patients who receive haplo-HSCT ${ }^{50}$. Outcomes with the TCR $\alpha \beta^{+} \mathrm{CD} 19^{+}$depleted haplo-HSCT approach in which no additional GvHD prophylaxis was employed appear promising in malignant and $\mathrm{NMD}^{3,23,47}$. Clinical results with CD45RA ${ }^{+} \mathrm{TCD}$ in the context of haplo-HSCT are so far limited ${ }^{48}$. Our PID results using CD45RA ${ }^{+}$TCD grafts are poor and insufficient to provide useful information. Further studies will be needed to further define the role of this approach in NMD. 
Immune reconstitution in the early post-transplant phase, in patients who received haplo-HSCT, relies on the peripheral expansion of graft-infused $\mathrm{T}$ cells ${ }^{49}$. The pretransplant use of serotherapy has also a strong impact on immune reconstitution by inducing in vivo $\mathrm{TCD}^{50}$. It has been shown that the absolute lymphocyte counts before ATG and alemtuzumab infusions and the day on which serotherapy is initiated influence early immune reconstitution ${ }^{51}$. We observed an important delay in the immune recovery in our cohort of patients. It took 6 months to have more than $200 \mathrm{CD} 4^{+}$cells/ $\mu$ land significance B lymphocytes. However, our reconstitution data is limited, because it was not routinely performed at the determined analysis times. The slowly immune reconstitution might impact the high TRM at day 100 as well as the outcomes of bacterial infections or viral reactivations.

This study presents several limitations. First, it is retrospective in nature and was performed in hospitals with a variable level of experience in these types of protocols. NMDs are a heterogeneous group of diseases with a diverse physiopathology that makes difficult the selection of the type of graft as well as the type of coding and GvHD prophylaxis. However, these results allow us to provide knowledge to design future strategies to improve the results of the haplo-HSCT, allowing this to be a more feasible option in patients with NMDs. Besides, many centers performed haplo-HSCT for a low number of patients which makes it difficult to overcome the learning curve needed for this type of transplant in these particular diseases; nevertheless it is possible to recommend moving on to successful platforms reported as PT-Cy and TCR $\alpha \beta^{+}$ depletion in NMD pediatric patients. Either platform should be chosen depending on the comfort level of the center. Nevertheless, ex vivo depletion should not be recommended in all centers, and be limited to only those centers where the experience, technical and logistical capabilities are available.

In conclusion, the haplo-HSCT approaches in NMDs are necessary but more studies are needed to propose a future strategy because of the great variability in the patients' underlying diseases, comorbidities, and appropriate conditioning regimens. The choice between PT-Cy and TCD platforms in NMDs is challenging and studies on larger patient samples will help refine our decisions in the future. Besides, it would be recommended to centralize this rare procedure on experts' centers.

\section{DATA AVAILABILITY STATEMENT}

Data available on request from the authors

\section{CONFLICT OF INTEREST STATEMENT}

The authors declare that the research was conducted in the absence of any commercial or financial relationships that could be construed as a potential conflict of interest.

\section{ACKNOWLEDGMENTS}

We are indebted to all the patients who agreed to participate in this trial, as well as to the personnel of the hospital involved in the study for their support and data collection.

This work was supported in part by the National Health Service of Spain, Instituto de Salud Carlos III (ISCIII), FONDOS FEDER grant (FIS) PI18/01301, the CRIS Cancer Foundation (http://criscancer.org) and the Association Pablo Ugarte (http://www.asociacionpablougarte.es/).

\section{REFERENCES}

1. Shenoy S, Boelens JJ. Advances in unrelated and alternative donor hematopoietic cell transplantation for nonmalignant disorders. Curr Opin Pediatr . 2015;27(1):9-17. doi:10.1097/MOP.0000000000000179

2. Bielorai B, Jacoby E, Varda-Bloom N, Hutt D et al . Haploidentical hematopoietic stem cell transplantation with $\alpha \beta T C R+/ C D 19+$ depletion in pediatric patients with malignant and non-malignant disorders. Bone Marrow Transplant . 2019;54:694-697. doi:10.1038/s41409-019-0607-0

3. Bertaina A, Merli P, Rutella S, Pagliara D et al . HLA-haploidentical stem cell transplantation after removal of $\alpha \beta+\mathrm{T}$ and $\mathrm{B}$ cells in children with nonmalignant disorders. Blood . 2014;124(5):822-826. doi:10.1182/blood-2014-03-563817 
4. Uppuluri R, Sivasankaran M, Patel S, Swaminathan VV et al . Haploidentical Stem Cell Transplantation in Children with Benign Disorders: Improved Survival and Cost-Effective Care Over 15 Years from a Single Center in India. Indian J Hematol Blood Transfus . 2019;35(3):426-430. doi:10.1007/s12288-019-01087-9

5. Kwon M, Bautista G, Balsalobre P, Sánchez-Ortega I et al . Haplo-Cord transplantation compared to haploidentical transplantation with post-transplant cyclophosphamide in patients with AML. Bone Marrow Transplant . 2017;52(8):1138-1143. doi:10.1038/bmt.2017.36

6. Tan EY, Boelens JJ, Jones SA, Wynn RF. Hematopoietic Stem Cell Transplantation in Inborn Errors of Metabolism. Front Pediatr . 2019;7:433. doi:10.3389/fped.2019.00433

7. Ciurea SO, Al Malki MM, Kongtim P, Fuchs EJ et al. The European Society for Blood and Marrow Transplantation (EBMT) consensus recommendations for donor selection in haploidentical hematopoietic cell transplantation. Bone Marrow Transplant . 2020;55(1):12-24. doi:10.1038/s41409-019-0499-z

8. Castagnoli R, Delmonte OM, Calzoni E, Notarangelo LD. Hematopoietic Stem Cell Transplantation in Primary Immunodeficiency Diseases: Current Status and Future Perspectives. Front Pediatr . 2019;7. doi:10.3389/fped.2019.00295

9. Reisner Y, Hagin D, Martelli MF. Haploidentical hematopoietic transplantation: current status and future perspectives. Blood . 2011;118(23):6006-6017. doi:10.1182/blood-2011-07-338822

10. Moretta L, Locatelli F, Pende D, Marcenaro E et al . Killer Ig-like receptor-mediated control of natural killer cell alloreactivity in haploidentical hematopoietic stem cell transplantation. Blood . 2011;117(3):764771. doi:10.1182/blood-2010-08-264085

11. Baumeister SHC, Rambaldi B, Shapiro RM, Romee R. Key Aspects of the Immunobiology of Haploidentical Hematopoietic Cell Transplantation.Front Immunol . 2020;11(February):1-26. doi:10.3389/fimmu.2020.00191

12. Im HJ, Koh KN, Choi ES, Jang S et al . Excellent outcome of haploidentical hematopoietic stem cell transplantation in children and adolescents with acquired severe aplastic anemia. Biol Blood Marrow Transplant . 2013;19(5):754-759. doi:10.1016/j.bbmt.2013.01.023

13. Im HJ, Koh KN, Seo JJ. Recent advances in haploidentical hematopoietic stem cell transplantation using ex vivo T cell-depleted graft in children and adolescents. Blood Res . 2016;51(1):8-16. doi:10.5045/br.2016.51.1.8

14. Klein OR, Chen AR, Gamper C, Loeb D et al . Alternative-Donor Hematopoietic Stem Cell Transplantation with Post-Transplantation Cyclophosphamide for Nonmalignant Disorders. Biol Blood Marrow Transplant . 2016;22(5):895-901. doi:10.1016/j.bbmt.2016.02.001

15. Oevermann L, Schulte JH, Hundsdörfer P, Hakimeh D et al . HLA-haploidentical hematopoietic stem cell transplantation in pediatric patients with hemoglobinopathies: current practice and new approaches.Bone Marrow Transplant . 2019;54(S2):743-748. doi:10.1038/s41409-019-0598-x

16. Zahid MF, Rizzieri DA. Haploidentical Hematopoietic Stem Cell Transplantation: Expanding the Horizon for Hematologic Disorders. Adv Hematol . 2016;2016:1-8. doi:10.1155/2016/1423493

17. Diaz MA, Pérez-Martínez A, Herrero B, Deltoro N et al . Prognostic factors and outcomes for pediatric patients receiving an haploidentical relative allogeneic transplant using CD3/CD19-depleted grafts. Bone Marrow Transplant . 2016;51(9):1211-1216. doi:10.1038/bmt.2016.101

18. Federmann B, Bornhauser M, Meisner C, Kordelas L et al . CD3/CD19 depletion and reduced intensity conditioning: A phase II study.Haematologica . 2012;97(10):1523-1531. doi:10.3324/haematol.2011.059378

19. Or-Geva N, Reisner Y. The evolution of T-cell depletion in haploidentical stem-cell transplantation. $\mathrm{Br}$ J Haematol . 2016;172(5):667-684. doi:10.1111/bjh.13868 
20. Airoldi I, Bertaina A, Prigione I, Zorzoli A, et al . Gammadelta T cell reconstitution after HLAhaploidentical hematopoietic transplantation depleted of TCR-alphabeta+/ CD19 + lymphocytes.Blood . 2015;125(15):2349-2358. doi:10.1182/blood-2014-09-599423.I.A.

21. Gennery AR, Slatter MA, Grandin L, Taupin P et al.Transplantation of hematopoietic stem cells and long-term survival for primary immunodeficiencies in Europe: Entering a new century, do we do better? $J$ Allergy Clin Immunol . 2010;126(3):602-610.e11. doi:10.1016/j.jaci.2010.06.015

22. Pai S-Y, Logan BR, Griffith LM, Buckley RH et al . Transplantation Outcomes for Severe Combined Immunodeficiency, 2000-2009. N Engl J Med . 2014;371(5):434-446. doi:10.1056/NEJMoa1401177

23. Bertaina A, Zecca M, Buldini B, Sacchi N et al . Unrelated donor vs HLA-haploidentical a/b T-celland B-cell-depleted HSCT in children with acute leukemia. Blood . 2018;132(24):2594-2607.

24. Erbey F, Akçay A, Atay D, Ovalı E, Öztürk G. Comparison of outcomes after HLA-matched unrelated and $\alpha \beta$ T-cell-depleted haploidentical hematopoietic stem cell transplantation for children with high-risk acute leukemia. Pediatr Transplant . 2018 Jun;22(4):e13192. doi: 10.1111/petr.13192

25. Locatelli F, Merli P, Pagliara D, Li Pira G et al . Outcome of children with acute leukemia given HLAhaploidentical HSCT after ab T-cell and B-cell depletion. Blood . 2017;130(5):677-685. doi:10.1182/blood2017-04-779769

26. González-Llano O, González-López EE, Marcos-Ramírez ER, Gómez-Almaguer D, et al . Haploidentical peripheral blood stem cell transplantation with posttransplant cyclophosphamide in children and adolescents with hematological malignancies. 2016;(October 2015):2033-2037. doi:10.1002/pbc.26131

27. Escudero A, Martínez-Romera I, Fernández L, Valentin J et al . Donor KIR Genotype Impacts on Clinical Outcome after T Cell-Depleted HLA Matched Related Allogeneic Transplantation for High-Risk Pediatric Leukemia Patients. Biol Blood Marrow Transplant . 2018;24(12):2493-2500. doi:10.1016/j.bbmt.2018.08.009

28. Pérez-Martínez A, Ferreras C, Pascual A, Gonzalez-Vicent M et al . Haploidentical transplantation in high-risk pediatric leukemia: A retrospective comparative analysis on behalf of the Spanish working Group for bone marrow transplantation in children (GETMON) and the Spanish Grupo for hematopoietic transplantation (GETH). Am J Hematol . 2020;95(1):28-37. doi:10.1002/ajh.25661

29. Sisinni L, Gasior M, de Paz R, Querol S et al . Unexpected High Incidence of Human Herpesvirus-6 Encephalitis after Naive T Cell-Depleted Graft of Haploidentical Stem Cell Transplantation in Pediatric Patients. Biol Blood Marrow Transplant . 2018;24(11):2316-2323. doi:10.1016/j.bbmt.2018.07.016

30. Carpenter PA, BS MBs and MLMM. Management of Acute Graft Versus Host Disease in Children. Pediatr Clin North Am . 2010;57(1):273-295. doi:10.1038/mp.2011.182.doi

31. Jagasia MH, Greinix HT, Arora M, Williams KM et al . National Institutes of Health Consensus Development Project on Criteria for Clinical Trials in Chronic Graft-versus-Host Disease: I. The 2014 Diagnosis and Staging Working Group Report. 2015;21(3):389-401. doi:10.1016/j.bbmt.2014.12.001.National

32. Holtan SG, DeFor TE, Lazaryan A, Bejanyan N et al . Composite end point of graft-versus-host diseasefree, relapse-free survival after allogeneic hematopoietic cell transplantation. Blood . 2015;125(8):1333-1338. doi:10.1182/blood-2014-10-609032

33. Gray RJ. A Class of K-Sample Tests for Comparing the Cumulative Incidence of a Competing Risk. Ann Stat . 1988;16(3):1141-1154. doi:10.1214/aos/1176350951

34. Zecca M, Strocchio L, Pagliara D, Comoli P et al . HLA-haploidentical T cell-depleted allogeneic hematopoietic stem cell transplantation in children with fanconi anemia. Biol Blood Marrow Transplant . 2014;20(4):571-576. doi:10.1016/j.bbmt.2014.01.015

35. Bahr TL, Lund T, Sando NM, Orchard PJ, Miller WP. Haploidentical transplantation with posttransplant cyclophosphamide following reduced-intensity conditioning for osteopetrosis: Outcomes in three 
children. Bone Marrow Transplant . 2016;51(11):1546-1548. doi:10.1038/bmt.2016.180

36. Balashov D, Shcherbina A, Maschan M, Trakhman P et al . Single-Center Experience of Unrelated and Haploidentical Stem Cell Transplantation with TCR $\alpha \beta$ and CD19 Depletion in Children with Primary Immunodeficiency Syndromes. Biol Blood Marrow Transplant . 2015;21(11):1955-1962. doi:10.1016/j.bbmt.2015.07.008

37. Cao LQ, Xu LP, Zhang XH, et al. Relationship of Cell Compositions in Allografts with Outcomes after Haploidentical Transplantation for Acquired Severe Aplastic Anemia: Effects of CD34+ and CD14+ Cell Doses.Chin Med J (Engl) . 2018;131(18):2185-2192. doi:10.4103/0366-6999.240810

38. Choi ES, Im HJ, Kim H, Koh KN et al . Depletion of $\alpha \beta+\mathrm{T}$ cells for a haploidentical hematopoietic stem cell transplantation in children. J Clin Apher . 2018;33(4):521-528. doi:10.1002/jca.21634

39. Esteves I, Bonfim C, Pasquini R, Funke V et al . Haploidentical BMT and post-transplant Cy for severe aplastic anemia: A multicenter retrospective study. Bone Marrow Transplant . 2015;50(5):685-689. doi:10.1038/bmt.2015.20

40. Shah RM, Elfeky R, Nademi Z, Qasim W et al . T-cell receptor $\alpha \beta+$ and CD19+ cell-depleted haploidentical and mismatched hematopoietic stem cell transplantation in primary immune deficiency. J Allergy Clin Immunol . 2018;141(4):1417-1426.e1. doi:10.1016/j.jaci.2017.07.008

41. Freeman AF. Hematopoietic Stem Cell Transplantation in Primary Immunodeficiencies beyond Severe Combined Immunodeficiency. J Pediatric Infect Dis Soc . 2018;7:S79-S82. doi:10.1093/jpids/piy114

42. Wahadneh AM, Dahman HA Bin, Abu Shukear ME, et al . Mismatched Related Hematopoietic Stem Cell Transplantation in Primary Immunodeficiency. Saudi J Kidney Dis Transpl . 2013 Vol 24(6):1137-43 doi: 10.4103/1319-2442.121269.

43. Kohli S, Rastogi N, Nivargi S, Thakkar D, Katewa S, Yadav SP. Successful Haploidentical Stem Cell Transplant With Posttransplant Cyclophosphamide for Hemophagocytic Lymphohistiocytosis. J Pediatr Hamatol Oncol. 2019 Apr;41(3):e158-e160. doi: 10.1097/MPH.0000000000001265.

44. Neven B, Diana JS, Castelle M, Magnani A et al . Haploidentical Hematopoietic Stem Cell Transplantation with Post-Transplant Cyclophosphamide for Primary Immunodeficiencies and Inherited Disorders in Children. Biol Blood Marrow Transplant . 2019;25(7):1363-1373. doi:10.1016/j.bbmt.2019.03.009

45. Cheng $\mathrm{Y}, \mathrm{Xu} \mathrm{Z}$, Zhang $\mathrm{Y}, \mathrm{Wu} \mathrm{J}$ et al . First-line choice for severe aplastic anemia in children: Transplantation from a haploidentical donor vs immunosuppressive therapy. Clin Transplant . 2018;32(2). doi:10.1111/ctr.13179

46. Yue Y, Song Z, Li J, Feng S, Liu R, Shi X. Posttransplant Cyclophosphamide for HLA-Haploidentical Transplantation in Patients With Mucopolysaccharidosis. Vol 40.; 2018. www.jpho-online.com.

47. Slatter M, Nademi Z, Leahy TR, Sykora K-W et al . Haploidentical CD3 TCRalphabeta and CD19depleted second stem cell transplant for steroid-resistant acute skin graft versus host disease.J Allergy Clin Immunol . 2016;138(2):603-605.e1. doi:10.1016/j.jaci.2015.12.1335

48. Schulz AS, Classen CF, Mihatsch WA, Sigl-Kraetzig M et al . HLA-Haploidentical Blood Progenitor Cell Transplantation in Osteopetrosis. Blood 2002. 99(9):3458-346.

49. Xu LP, Wang SQ, Wu DP, Wang JM et al . Haplo-identical transplantation for acquired severe aplastic anaemia in a multicentre prospective study. Br J Haematol . 2016;175(2):265-274. doi:10.1111/bjh.14225

50. Admiraal R, van Kesteren C, Jol-van der Zijde CM, Lankester ACet al . Association between antithymocyte globulin exposure and $\mathrm{CD} 4+$ immune reconstitution in paediatric haemopoietic cell transplantation: a multicentre, retrospective pharmacodynamic cohort analysis. Lancet Haematol . 2015;2(5):e194-e203. doi:10.1016/S2352-3026(15)00045-9 
51. Willemsen L, Jol-van der Zijde CM, Admiraal R, Putter H et al . Impact of Serotherapy on Immune Reconstitution and Survival Outcomes After Stem Cell Transplantations in Children: Thymoglobulin Versus Alemtuzumab. Biol Blood Marrow Transplant . 2015;21(3):473-482. doi:10.1016/j.bbmt.2014.11.674

Figure 1. A) OS, GRFS and GF of PID and others NMD; B) OS, GRFS and GF of previous HSCT vs first haplo-HSCT.

Supplemental Figure 1. Recovery of $\mathrm{CD} 3^{+}, \mathrm{CD} 4^{+}, \mathrm{CD} 8^{+}, \mathrm{CD} 16^{+} \mathrm{CD} 56^{+}$and $\mathrm{CD} 19^{+}$cells in PIDs and other NMDs at 30,60, 80, 180 and 360 days after transplantation.

\section{Hosted file}

Table 1.docx available at https://authorea.com/users/334925/articles/460870-haploidenticaltransplantation-in-pediatric-non-malignant-diseases-a-retrospective-analysis-on-behalfof-the-spanish-group-for-hematopoietic-transplantation-geth

\section{Hosted file}

TABLE 2.docx available at https://authorea.com/users/334925/articles/460870-haploidenticaltransplantation-in-pediatric-non-malignant-diseases-a-retrospective-analysis-on-behalfof-the-spanish-group-for-hematopoietic-transplantation-geth
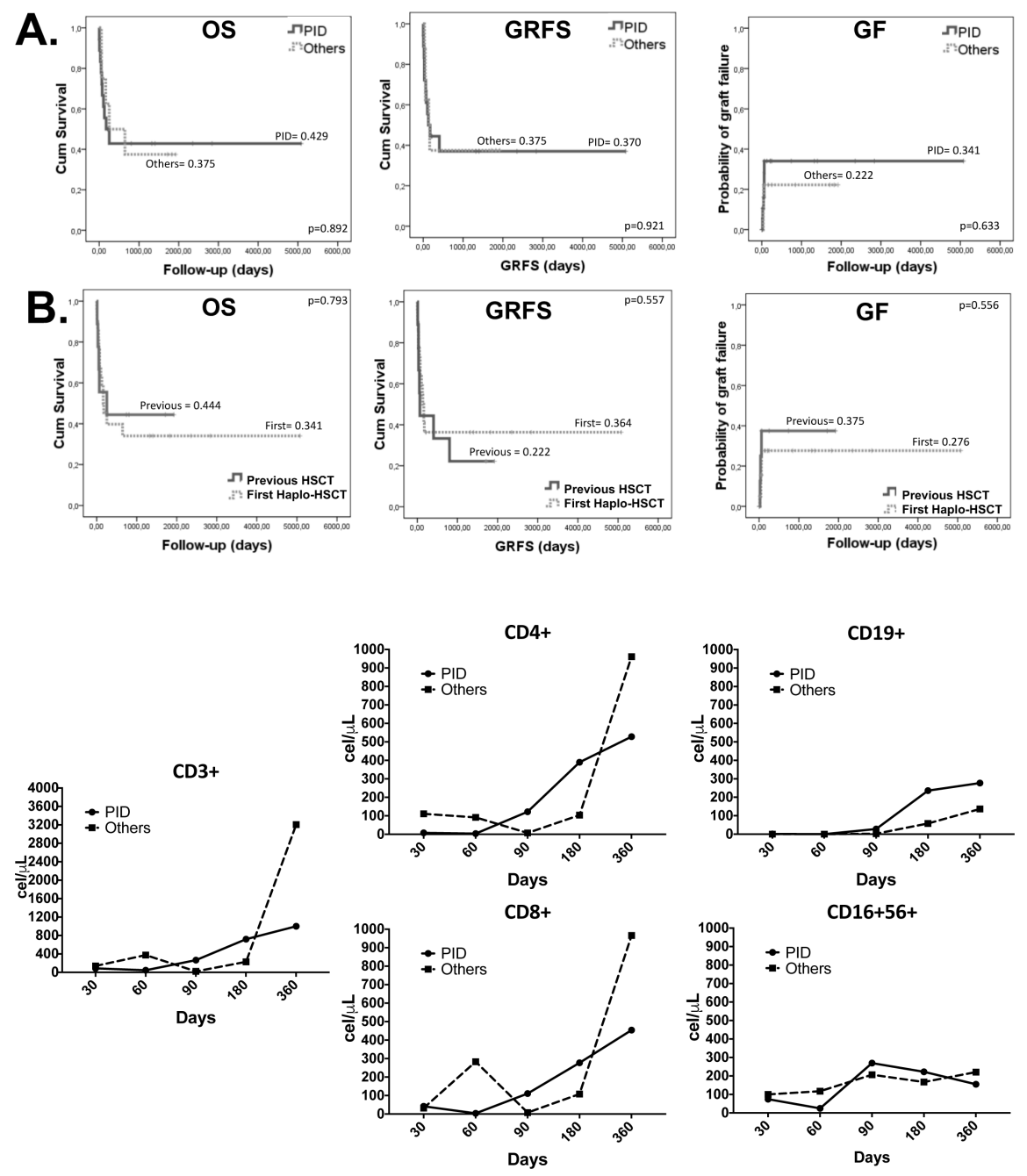